\title{
A Novel Heterogeneous Approximate Multiplier for Low Power and High Performance
}

\author{
Ihsen Alouani $^{\circledR}$, Hamzeh Ahangari, Ozcan Ozturk, and Smail Niar
}

\begin{abstract}
Approximate computing is a design paradigm considered for a range of applications that can tolerate some loss of accuracy. In fact, the bottleneck in conventional digital design techniques can be eliminated to achieve higher performance and energy efficiency by compromising accuracy. In this letter, a new architecture that engages accuracy as a design parameter is presented, where an approximate parallel multiplier using heterogeneous blocks is implemented. Based on design space exploration, we demonstrate that introducing diverse building blocks to implement the multiplier rather than cloning one building block achieves higher precision results. We show experimental results in terms of precision, delay, and power dissipation as metrics and compare with three previous approximate designs. Our results show that the proposed heterogeneous multiplier achieves more precise outputs than the tested circuits while improving performance and power tradeoffs.
\end{abstract}

Index Terms-Adders, approximate computing, multiplying circuits.

\section{INTRODUCTION}

W ITH the increase in the amount of data and complexity of tasks supported by battery-operated electronic devices, there is a continuous for design techniques to conserve power consumption, while achieving the desired performance. In fact, new generations of embedded systems are designed to process power hungry applications that handle heavy workloads. For example, in mobile devices, systems need to process multimedia content, recognize patterns, and interact intelligently with their environment. This trend impacts directly the computing paradigm due to the new specific demands in applications which are not necessarily aiming at a precise numerical result; instead, they try to achieve a sufficient quality of results. Therefore, digital signal processing (DSP) has become one of the most attractive topics in semiconductor industry in the past 30 years. According to previous studies [16], the global market share of DSP architectures exceeds $95 \%$ of the total volume of processors sold. A wide range of multimedia applications, such as image, voice and video processing, data searching,

Manuscript received June 15, 2017; accepted November 17, 2017. Date of publication November 28, 2017; date of current version June 6, 2018. This manuscript was recommended for publication by S. Parameswaran. (Corresponding author: Ihsen Alouani.)

I. Alouani and S. Niar are with the LAMIH Lab, University of Valenciennes, 59300 Valenciennes, France (e-mail: ihsen.alouani@univ-valenciennes.fr; smail.niar@univ-valenciennes.fr).

H. Ahangari and O. Ozturk are with the Department of Computer Engineering, Bilkent University, 06800 Ankara, Turkey (e-mail: hamzeh@bilkent.edu.tr; ozturk@cs.bilkent.edu.tr).

Color versions of one or more of the figures in this paper are available online at http://ieeexplore.ieee.org.

Digital Object Identifier 10.1109/LES.2017.2778341 recognition, etc., are highly tolerant to errors and their quality of service is not affected by a certain amount of precision loss. Chippa et al. [6] analyzed a benchmark suite of 12 recognition, mining, and search applications and found that on average, $83 \%$ of the runtime computations can tolerate at least some degree of approximation. Hence, for this type of applications, there is a change in design methodology toward approximate computing rather than the classical accurate computing design. Approximate computing relies on the range of tolerated inaccuracy in the computational process to improve power efficiency and performance.

\section{RELATED WORK}

To reduce power consumption of CMOS circuits, a commonly used approach is to aggressively scale supply voltage beyond the nominal value. However, this technique has considerably negative drawbacks on the quality of service and leads to a degradation in terms of performance. While algorithmic noise tolerance schemes [15] are meant to compensate this degradation, the new circuits already have very low voltages and are no longer allow systematic use of this technique.

Previous works proposed reducing combinational circuit complexity through approximate computing systems. The main objective is to design circuits with lower number of transistors leading to a reduction in delay and power consumption. A reduction in circuit complexity at transistor level in an adder circuit provides a more important reduction in power consumption compared to the conventional low power design techniques [10]. Shin and Gupta [14] proposed a logic synthesis approach to design circuits for implementing approximate functions by considering error rate (ER) as metric for accuracy.

As one of the key components in arithmetic circuits, many approximation schemes of adder implementations were proposed. Segmented adders are implemented in [11]-[13] by several smaller adders operating in parallel, where the carry propagation sequence is truncated into shorter segments. Another method for reducing the critical path delay and power dissipation of a conventional combinational circuit is by approximating their elementary full adder blocks [7]-[10].

While adders have been extensively studied, there has been relatively less work in the literature that focus on approximate multipliers. In [3], approximate partial products are computed based on approximate 2 by 2 elementary multipliers, while a tree of accurate adders is used to accumulate the elementary products. Huang et al. [4] studied the 


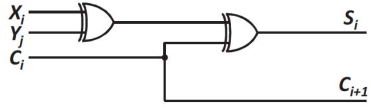

(a)

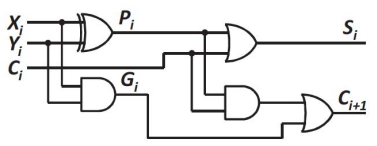

(c)

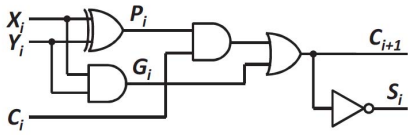

(e)

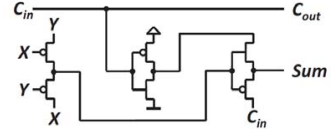

(b)

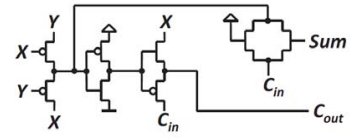

(d)

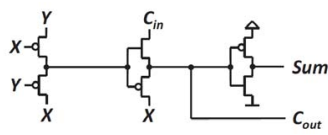

(f)
Fig. 1. Three considered inexact adder cells proposed in [1]. Logic diagrams of (a) InXA1, (c) InXA2, and (e) InXA3. Transistor circuit diagrams of (b) InXA1, (d) InXA2, and (f) InXA3.

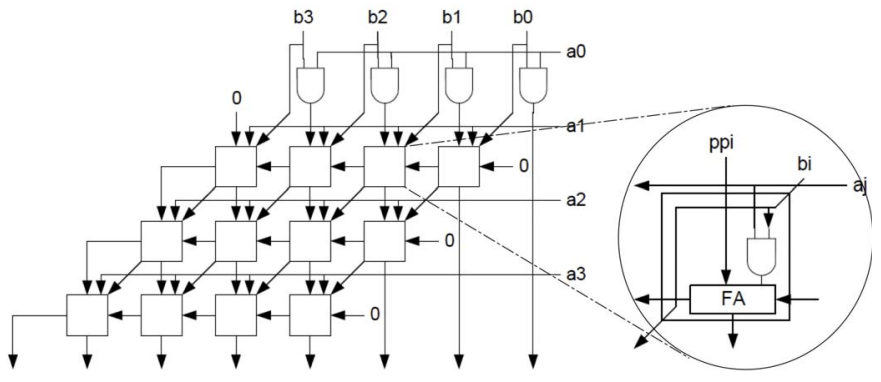

Fig. 2. $4 \times 4$ multiplier architecture.

implementation of approximate adders for the final stage addition in a multiplier design. Kyaw et al. [5] proposed the error tolerant multiplier which suggests splitting the multiplier into an accurate multiplication part for MSBs and a nonaccurate multiplication part for LSBs.

All of these works consider a homogeneous design pattern and rely on a single implementation of approximate elements to build their circuits. In this letter, we propose a new approximate circuit design methodology in which we consider a set of different adder blocks to build a heterogeneous multiplier. We use the three approximate implementations of full adders proposed by [1] shown in Fig. 1 as possible adder implementations and explore the design space to converge to an optimal heterogeneous design.

\section{Proposed Architecture}

In this letter, we use a new methodology to build approximate circuits for a heterogeneous approximate multiplier. We propose to rely on a set of different inexact elementary blocks instead of one block that is replicated to build the desired circuit. The purpose is to take advantage of the design flexibility given by diversifying the approximate elements to comprehensively benefit from the error masking mechanisms; thereby reducing precision loss. More specifically, the logical masking mechanism is applied, where an error propagates to reach a gate's input while another input is in controlling state (for

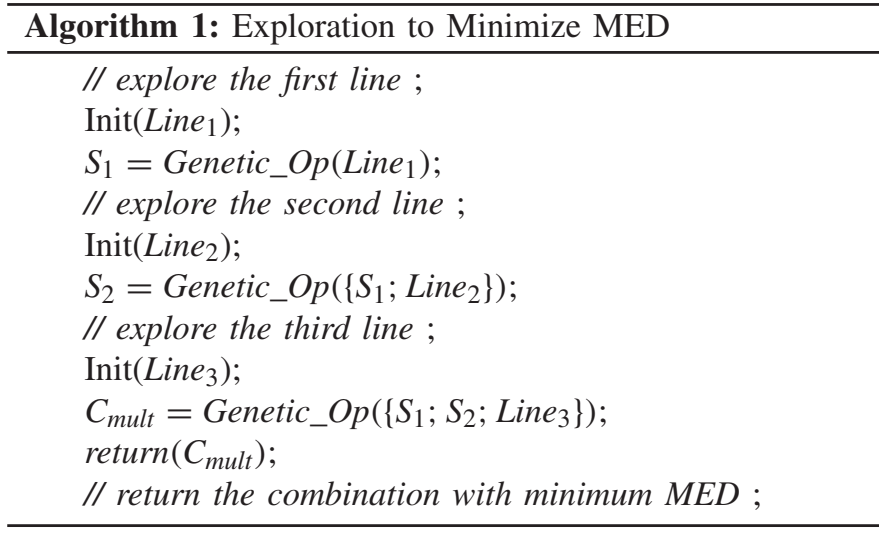

example, a "0" input of a AND gate). Hence, the idea is to explore, at design time, the set of inexact implementations to identify those with the minimum number of errors propagating through the circuit to the output. This way, we increase the precision of the overall circuit. Therefore, we proceed to a design space exploration phase in order to select the most accurate design combination.

Algorithm 1 details the design space exploration phase. The objective is to find the combination of full adders that minimizes the mean error distances (MEDs) for the overall design, where MED is the average arithmetic deviation from the accurate design. The idea is based on genetic algorithm (GA) exploration algorithm and adapted to our design problem. The exploration process is achieved in three steps corresponding to the architecture full adders lines. The first step lunches GA exploration on the first line full adders (see Fig. 2) with considering the remaining subsequent blocs as exact circuits. The result of this step is a set of implementations with the lowest MED that we refer to as $S_{1}$. In the next step, we iterate the exploration with the first and the second lines with an exact implementation of the third line. The second line is explored based on GA while the considered exploration space of the first line is $S_{1}$. From this step we extract a new set of best implementations that we refer to as $S_{2}$. Finally, the same process is applied for the whole circuit to get the overall best implementation.

\section{Precision Evaluation}

To assess the precision of our architecture, simulations are pursued and results are compared with the previous approximate designs. The following performance metrics are used for evaluation purposes.

1) Error Distance: Error distance (ED) is the arithmetic difference between the exact result $R^{*}$ and the approximate result $R$, that is

$$
\mathrm{ED}=\left|R^{*}-R\right| .
$$

2) Mean Relative Error Distance: Mean relative error distance (MRED) is the average of the relative ED, where RED is given by

$$
\mathrm{RED}=\frac{\mathrm{ED}}{R^{*}}
$$




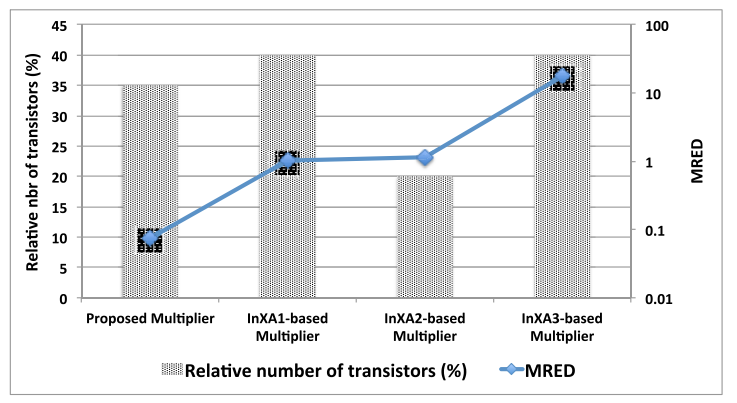

Fig. 3. MRED compared with previous homogeneous approximate multipliers.

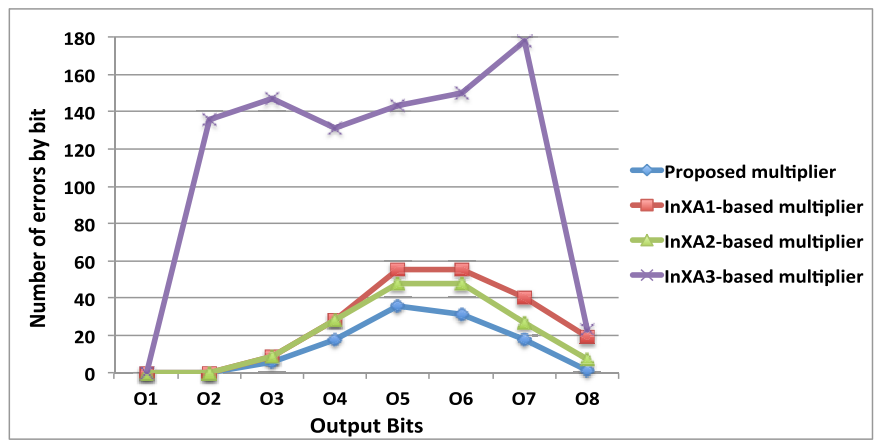

Fig. 4. Distribution of ED within the minimal-error circuits compared to the InXA1, InXA2, and InXA3-based multiplier.

Fig. 3 shows the results in terms of mean relative error (MRED) along with the relative number of transistors (\%) compared with respect to the exact implementation and Fig. 4 shows the number of errors by output bit. While the implementation of the proposed multiplier costs only $35 \%$ of the number of transistors used in the exact multiplier, it achieves the lowest MRED, thereby achieving the most accurate results compared with the homogeneous implementations.

\section{Performance and Energy Evaluation}

In this section, we compare the proposed approximate multiplier with the previously proposed schemes at $45 \mathrm{~nm}$ with PTM [18] using Advanced Design System simulation platform. All input combinations are tested exhaustively and the results in terms of delay and energy are shown in Figs. 5 and 6 for the average and the worst case.

As shown in these figures, the proposed multiplier outperforms InXA2- and InXA3-based approximate multipliers in terms of performance metric. Even though there is a delay overhead compared to InXA1-based circuit, InXA1based multiplier consumes $24 \%$ more energy compared to our multiplier.

\section{Approximate Computing Applications}

\section{A. Edge Detection Through Sobel Filter}

The Sobel operator, or Sobel filter, is a widely used tool in image processing and computer vision applications, particularly within edge detection algorithms where it emphasizes the edges of a grayscale image. Technically, it consists of a discrete differentiation operator, that approximates the gradient of the image intensity function. At each pixel, the result

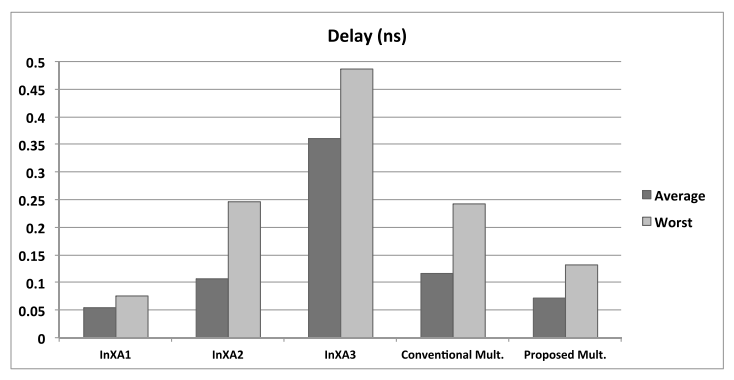

Fig. 5. Average and worst case delay for previously proposed approximate multipliers, our multiplier, and conventional multiplier.

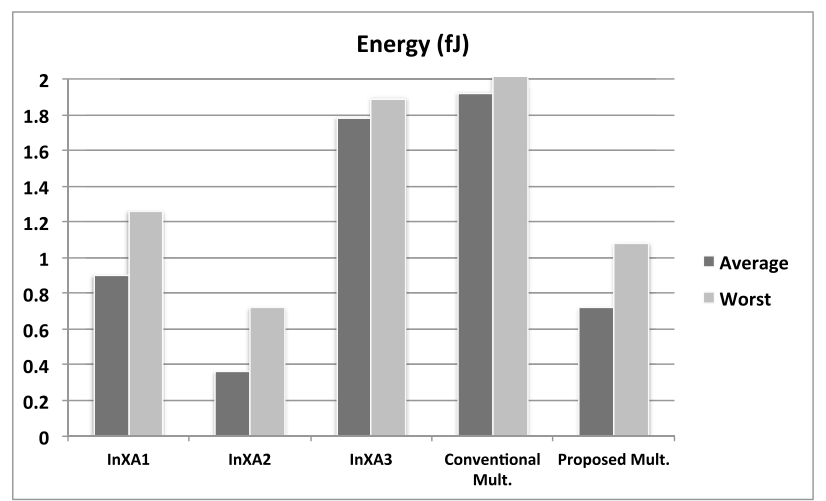

Fig. 6. Average and worst case energy dissipation for previous, proposed approximate multipliers and conventional multiplier.

TABLE I

MSE COMPARED TO EXACT FILTER

\begin{tabular}{|c|r|c|r|}
\hline Proposed Filter & InXA1-based & InXA2-based & InXA3-based \\
\hline 9.76 & 21.32 & 18.76 & 52.67 \\
\hline
\end{tabular}

of the Sobel filter can be the corresponding gradient vector or the norm of the bidirectional gradient. The Sobel operator is based on convolving the image with a small, separable, and integer-valued matrix in the $X$ and $Y$ directions.

We use the mean square error (MSE) metric to assess the quality of the different approximate filters compared to the reference output achieved by the exact filter as shown in Fig. 8 . The results shown in Table I demonstrate that the proposed multiplier is much more accurate in the Sobel operator implementation compared to the homogeneous implementations. It clearly has a lower MSE, which means that the deviation from the reference output is very low.

\section{B. K-Means-Based Clustering}

K-means clustering algorithm aims to partition $n$ observations into $k$ clusters in which each observation belongs to the cluster with the nearest mean, serving as a prototype of the cluster. We implement $k$-means for the Fisher Iris dataset using: exact multiplier, the proposed multiplier, and the three homogeneous multipliers. As shown in Fig. 7, the proposed approximate multiplier-based implementation achieves better results with only one erroneous classification case out of 150 


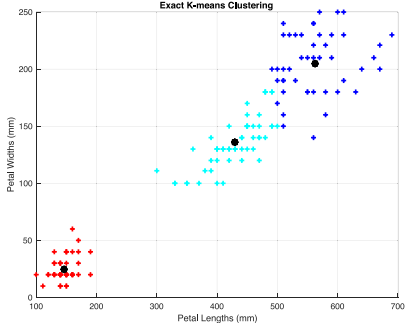

(a)

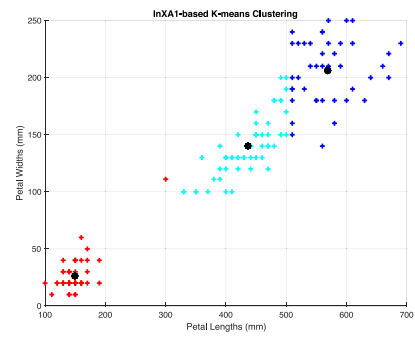

(c)

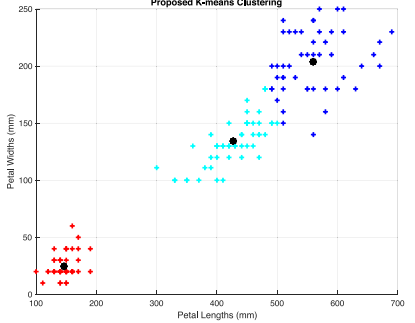

(b)

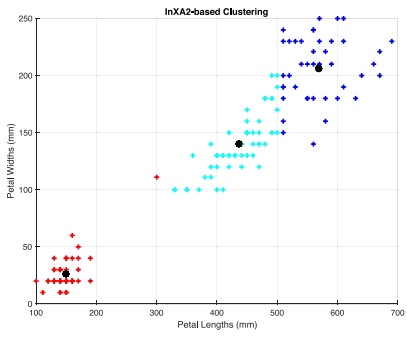

(d)

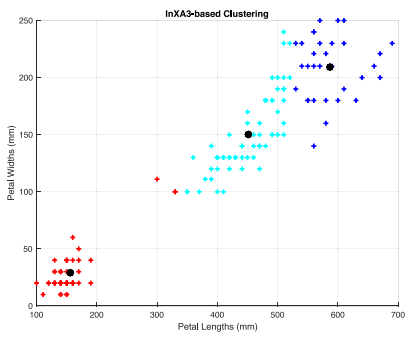

(e)

Fig. 7. $K$-means clustering results. (a) Exact $K$-means clustering. (b) $K$-means clustering with the proposed multiplier. (c) $K$-means clustering with the InXA1-based multiplier. (d) $K$-means clustering with the InXA2-based multiplier. (e) $K$-means clustering with the InXA3-based multiplier.
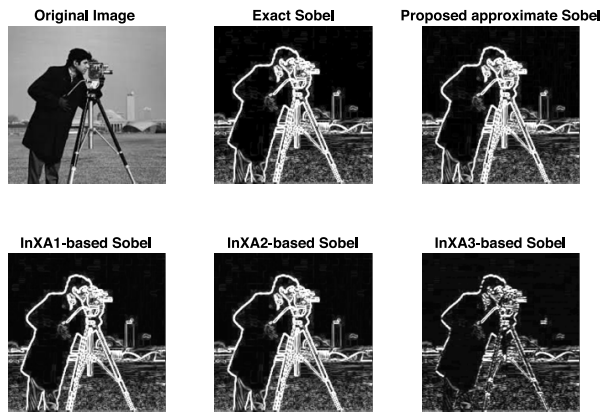

Fig. 8. Original image and the Sobel Filter implementations for: exact implementation, proposed multiplier, InXA1, InXA2, and InXA3.

observations compared with 5, 5, and 16 errors in InXA1-, InXA2-, and InXA3-based implementations, respectively.

\section{CONCLUSION}

In this letter, we propose a novel heterogeneous architecture that uses accuracy as a design parameter. Specifically, we build an approximate parallel multiplier based on different approximate implementations. After design space explorations, we realized that introducing different elementary architectures to implement the circuit leads to lower ERs compared to the classical homogeneous designs. In fact, the proposed design benefits from the masking mechanisms within logic elements in different cases to limit the overall deviation from the exact results. Our experiments show that the utilized design method results in an approximate multiplier with higher accuracy and better tradeoffs compared with previous circuits.

\section{REFERENCES}

[1] H. A. F. Almurib, T. N. Kumar, and F. Lombardi, "Inexact designs for approximate low power addition by cell replacement," in Proc. DATE Conf., Dresden, Germany, 2016, pp. 660-665.

[2] S.-L. Lu, "Speeding up processing with approximation circuits," Computer, vol. 37, no. 3, pp. 67-73, Mar. 2004

[3] P. Kulkarni, P. Gupta, and M. Ercegovac, "Trading accuracy for power with an underdesigned multiplier architecture," in Proc. 24th IEEE Int Conf. VLSI Design, Chennai, India, 2011, pp. 346-351.

[4] J. Huang, J. Lach, and G. Robins, "A methodology for energy-quality tradeoff using imprecise hardware," in Proc. Design Autom. Conf. (DAC), 2012, pp. 504-509.

[5] K. Y. Kyaw, W. L. Goh, and K. S. Yeo, "Low-power high-speed multiplier for error-tolerant application," in Proc. IEEE Int. Conf. Electron Devices Solid-State Circuits (EDSSC), 2010, pp. 1-4.

[6] V. K. Chippa, S. T. Chakradhar, K. Roy, and A. Raghunathan, "Analysis and characterization of inherent application resilience for approximate computing," in Proc. DAC, Austin, TX, USA, 2013, pp. 1-9.

[7] Z. Yang, A. Jain, J. Liang, J. Han, and F. Lombardi, "Approximate XOR/XNOR-based adders for inexact computing," in Proc. IEEE NANO, 2013, pp. 690-693.

[8] D. Nanu, Roshini P. K., D. Sowkarthiga, and K. S. A. Ameen, "Approximate adder design using CPL logic for image compression," Int. J. Innov. Res. Develop., vol. 3, no. 4, pp. 362-370, 2014.

[9] H. R. Mahdiani, A. Ahmadi, S. M. Fakhraie, and C. Lucas, "Bio-inspired imprecise computational blocks for efficient VLSI implementation of soft-computing applications," IEEE Trans. Circuits Syst. I, Reg. Papers, vol. 57, no. 4, pp. 850-862, Apr. 2010.

[10] V. Gupta, D. Mohapatra, A. Raghunathan, and K. Roy, "Low-power digital signal processing using approximate adders," IEEE Trans. Comput.-Aided Design Integr. Circuits Syst., vol. 32, no. 1, pp. 124-137, Jan. 2013.

[11] N. Zhu, W. L. Goh, and K. S. Yeo, "An enhanced low-power high-speed adder for error-tolerant application," in Proc. ISIC, 2009, pp. 69-72.

[12] D. Mohapatra, V. K. Chippa, A. Raghunathan, and K. Roy, "Design of voltage-scalable meta-functions for approximate computing," in Proc. DATE, 2011, pp. 1-6.

[13] A. B. Kahng and S. Kang, "Accuracy-configurable adder for approximate arithmetic designs," in Proc. DAC, 2012, pp. 820-825.

[14] D. Shin and S. K. Gupta, "Approximate logic synthesis for error tolerant applications," in Proc. Design Autom. Test Europe (DATE), 2010, pp. 957-960.

[15] R. Hegde and N. R. Shanbhag, "Soft digital signal processing," IEEE Trans. Very Large Scale Integr. (VLSI) Syst., vol. 9, no. 6, pp. 813-823, Dec. 2001.

[16] D. Liu, Embedded DSP Processor Design. Burlington, MA, USA: Morgan Kaufmann, 2008, p. 808.

[17] J. Liang, J. Han, and F. Lombardi, "New metrics for the reliability of approximate and probabilistic adders," IEEE Trans. Comput., vol. 62, no. 9, pp. 1760-1771, Sep. 2013.

[18] Predictive Technology Model (PTM) Website. Accessed: Dec. 2017. [Online]. Available: http://ptm.asu.edu 